\title{
Recto-Gluteal Fistula from Colonic Stent Migration and Perforation in a Patient with Metastatic Colorectal Cancer Treated with Folfox and Bevacizumab: A Case Report
}

\author{
Danielle Collins $^{1^{*}}$ and Eric J Dozois ${ }^{2}$ \\ ${ }^{1}$ Department of Surgery, Mayo Clinic Rochester, USA \\ ${ }^{2}$ Division of Colon \& Rectal Surgery, Mayo Clinic Rochester, USA
}

*Corresponding author: Danielle Collins, Department of Surgery, Mayo Clinic Rochester, 200 First Street SW Rochester, MN 55905, USA, E-mail: daniellecol@gmail.com

\section{Case Presentation}

A 47-year-old female with metastatic colorectal cancer underwent colonic stenting for an obstructing lesion at the recto-sigmoid junction. Staging CT imaging demonstrated a large bowel obstruction due to a stricturing lesion at the recto-sigmoid junction, numerous bilobar liver Mets and two lung nodules suspicious for metastatic disease. Colonoscopy and biopsy confirmed an adenocarcinoma $15 \mathrm{~cm}$ from the anal verge. She underwent an uneventful placement of a colonic self-expanding metal stent (SEMS) and then proceeded to undergo several cycles of systemic chemotherapy that included FOLFOX with bevacizumab. Three months later she developed severe pain in the right gluteal region and underwent a CT scan which demonstrated migration of the stent through the rectal wall, pelvic floor and gluteal musculature, creating a recto-gluteal fistula and $a b$ scess (Figure 1A and Figure 1B). She underwent incision and drainage of the gluteal abscess and formation of a transverse loop colostomy as an emergent procedure to control sepsis. One month later, she was referred to our institution for definitive surgical management. Following repeat imaging and multidisciplinary discussion with medical oncology, colorectal surgery and hepatobiliary surgery, a decision was made to perform an oncological colorectal resection with removal of the stent, reversal of the transverse loop colostomy, omental pedicle flap to the pelvis and construction of an end colostomy. The resected specimen (Figure 1C) showed a com- plete pathological response with negative lymph nodes (урT0, NO).

\section{Discussion}

The addition of bevacizumab to chemotherapeutic regimens for metastatic colorectal cancer has shown improved survival [1]. There is however a recognized bowel perforation rate of $1.9 \%$ in patients undergoing this treatment [2]. Recently, there has been some concern that there is a higher risk of perforation in patients who have an endoluminal stent placed for obstruction at the time of treatment with bevacizumab [3]. Imbulgoda, et al. retrospectively reviewed 87 patients who underwent SEMS placement with an overall perforation rate of $10.3 \%$. In this series, 2 out of 10 patients (20\%) treated with bevacizumab had a perforation [4]. Small, et al. reported-on stent complications in patients treated with bevacizumab ( $28 \mathrm{pts}$ ) and found a complication rate of $34.8 \%$, including a perforation frequency of $17.4 \%$ [5]. Treatment with bevacizumab nearly tripled the risk of perforation in their overall cohort. The mechanism of perforation is yet to be elucidated but generally occurs within 21 days to 3 months of initiation of the anti-angiogenic therapy. It has been postulated that tumor growth and then shrinkage associated with inhibition of angiogenesis leads to impaired healing and perforation which may be compounded by the radial forces exerted from a self-expanding stent. Interestingly, bevacizumab impairs wound healing overall and the inability to mount an inflammatory fibrotic response may play a role in the 

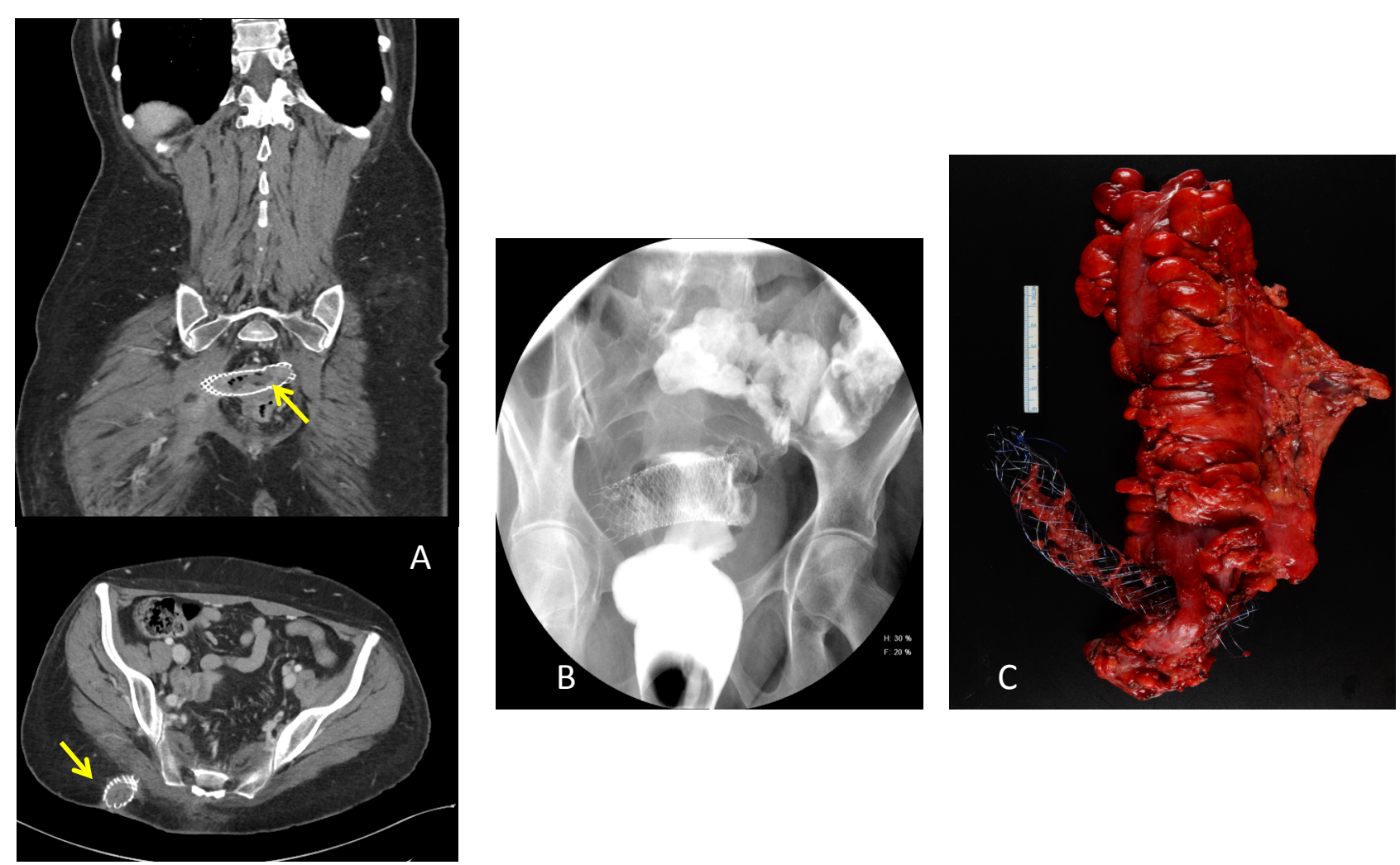

Figure 1: A) CT demonstrating the stent perforating horizontally through the rectum into the right gluteus (arrows);

B) Hypaque enema showing the stent position through the rectum; C) Post-resection image of the perforating rectal stent.

pathogenesis of a traumatic induced perforation. It is difficult to know exactly what the role of bevacizumab played in the complication from the stent in our patient. Given the perforation and dramatic migration of the stent, we strongly suspect that the combination of tumor regression, suppression of inflammatory response and delayed would healing induced by bevacizumab is likely responsible.

\section{Learning Point}

This case highlights the potential for serious complications in bevacizumab treated colorectal cancer patients who have a colonic stent and the importance of early investigation of symptoms that may be secondary to a stent complication. Although stenting is useful in the emergent setting to relieve colonic obstruction, perhaps a diverting ostomy may be safer in the long-term for patients who will receive bevacizumab-based chemotherapy. Ultimately, a discussion with medical oncology may be beneficial in formulating a treatment plan prior to inserting a colonic stent.

\section{Conflicts of Interest}

The authors have no conflicts of interest or financial disclosures.

\section{References}

1. Cao Y, Tan A, Gao F, Liu L, Liao C, et al. (2009) A meta-analysis of randomized controlled trials comparing chemotherapy plus bevacizumab with chemotherapy alone in metastatic colorectal cancer. Int J Colorectal Dis 24: 677685.

2. Kabbinavar FF, Flynn PJ, Kozloff M, Ashby MA, Sing A, et al. (2012) Gastrointestinal perforation associated with bevacizumab use in metastatic colorectal cancer: Results from a large treatment observational cohort study. Eur J Cancer 48: 1126-1132.

3. Fuccio L, Cennamo V (2010) Bevacizumab-based therapy and complication risk after colonic stent placement: Is it time for a warning? Gastrointest Endosc 72: 1330.

4. Imbulgoda A, MacLean A, Heine J, Drolet S, Vickers MM (2015) Colonic perforation with intraluminal stents and bevacizumab in advanced colorectal cancer: Retrospective case series and literature review. Can J Surg 58: 167-171.

5. Small AJ, Coelho Prabhu N, Baron TH (2010) Endoscopic placement of self-expandable metal stents for malignant colonic obstruction: Long-term outcomes and complication factors. Gastrointest Endosc 71: 560-572. 